\title{
Funcionalidad de las relaciones entre conceptos en la resolución de problemas
}

\section{Functionality of relationships among concepts for problem solving}

Carlos Emilio Reigosa Castro

IES Lucus Augusti y Facultad de Formación del Profesorado (Lugo, España)

carlosreigosa@edu.xunta.gal

RESUMEN • En este trabajo se analiza la evolución de las relaciones semánticas entre conceptos desarrolladas por un grupo de estudiantes de secundaria cuando se enfrentan en el laboratorio de Física a tareas prácticas concebidas como problemas. Los resultados indican que el proceso de desarrollar las relaciones semánticas para resolver las tareas fue un proceso laborioso en el que dichas relaciones van atravesando fases de distinta funcionalidad hasta llegar a ser herramientas útiles para la planificación de acciones, aunque en las tareas analizadas no se sigue siempre el mismo camino. Además, a la hora de que los estudiantes sean capaces de resolver una tarea, hemos visto que se superponen otros aspectos, como la presencia de eventuales dificultades epistemológicas y procedimentales.

PALABRAS CLAVE: Relaciones semánticas; Prácticas contextualizadoras; Laboratorio de física; Problemas abiertos; Indagación científica.

ABSTRACT - The evolution of the semantic relationships among concepts developed by a group of high school students while facing practical tasks conceived as problems in the Physics laboratory is analyzed in this paper. The results point that the process of developing the semantic relationships to solve the tasks was a laborious process in which the semantic relationships go through phases of different functionality until becoming useful tools for planning actions, although different paths were followed in the different analyzed tasks. Moreover, to get the students to be able to solve a task, we see that other aspects overlap, such as the presence of eventual epistemological and procedural difficulties.

KEYWORDS: Semantic relationships; Contextualyzing practices; Physics laboratory; Open problems; Scientific research. 


\section{INTRODUCCIÓN: OBJETIVOS Y FUNDAMENTACIÓN DEL TRABAJO}

La comprensión de la naturaleza de la actividad científica es un objetivo fundamental de la enseñanza de las ciencias, y, en lo que a esto respecta, es importante tener en cuenta que, como Wells (1999) indica, comprender las formas de actuar de una comunidad no puede ser independiente de la participación en las prácticas sociales en las que cobran sentido. Brown, Collins y Duguid (1989) consideran que el aprendizaje es un proceso de enculturación que se logra mediante la implicación progresiva en actividades similares a aquellas en las que participan los verdaderos miembros de la comunidad. Concretando para la comunidad científica, sus actividades tienen poco de aplicación mecánica de instrucciones y, de hecho, los científicos aprenden la cultura de la ciencia mediante la práctica y la inmersión en tareas complejas (Barab y Hay, 2001). A pesar de esto, en la enseñanza de las ciencias no procede excluir la importancia de la reflexión explícita sobre las prácticas científicas.

Puede parecer difícil que los estudiantes comprendan la naturaleza de la actividad científica (Bell, 2003), pero, por ejemplo, Metz (2004) muestra que los estudiantes pueden tener un éxito reseñable al ejecutar tareas científicas, aunque estas sean de cierta complejidad, y Stake y Mares (2001) aportan pruebas que indican que la implicación de los estudiantes en tareas de indagación científica hace mejorar su actitud ante la ciencia. Además, la indagación científica facilita el desarrollo de formas de pensar más sofisticadas (Charney et al., 2007), lo que aumenta sus habilidades de manejo de hipótesis, modelos, argumentos y conceptos. Kuhn et al. (2017) muestran que la implicación del alumnado en actividades científicas favorece las destrezas de investigación, análisis y argumentación, y para Richmond (1998) la inmersión en la cultura de la ciencia proporciona las oportunidades necesarias para ser testigos y participantes de la ciencia como actividad humana.

Un aspecto importante que hay que tener en cuenta a la hora de ayudar a los estudiantes a desarrollar una visión satisfactoria de la actividad científica es que los miembros de las comunidades se diferencian por las tareas que realizan y por las prácticas y recursos que ponen en juego, siendo centrales las prácticas discursivas (Roth y Bowen, 1995). En relación con esto, Cazden (1988) indica que toda institución social es un sistema de comunicación. El discurso ha sido definido como el lenguaje en uso en una comunidad (Lemke, 1997), de modo que la enculturación científica implica la apropiación del lenguaje de la ciencia. Apropiarse de ese lenguaje conlleva desarrollar un uso competente de sus recursos para crear significados, es decir, implica no solo un aprendizaje de su léxico o sintaxis, sino desarrollar un dominio de este en términos de sistema semiótico, por lo que los estudiantes deberían implicarse en procesos discursivos propios de la ciencia (Bjørkvold y Blikstad-Balas, 2018), perspectiva que asumimos en este estudio, teniendo en cuenta la relación básica entre discurso y acción.

El análisis que se muestra en este trabajo acerca de la creación de significados se realiza usando la noción de prácticas contextualizadoras de Lemke (1997). Para este autor, el significado de las palabras y acciones no está fijado unívocamente en estas, sino que es construido interaccionalmente por las personas. Las prácticas por medio de las cuales se llevan a cabo acciones o manifestaciones que tienen sentido para la comunidad son las prácticas de creación de significados o semióticas. Lemke (1997) considera que las acciones cobran significado al conectarlas a contextos. Los contextos sociales asociados con una acción en una comunidad particular son los contextos indexicales, término usado, por ejemplo, por Godino et al. (2012) para analizar la visualización en la educación matemática. Lo importante en la contextualización indexical son las pautas de acciones, las relaciones sistemáticas de acciones, que cobran sentido en relación con los contextos externos a los que hacen referencia. Un caso especial de contexto indexical son los contextos o patrones temáticos, referidos a cómo se habla en una comunidad determinada acerca de un tema concreto. En los contextos temáticos, las relaciones semánticas entre los conceptos son reconstruidas continuamente. 
Analizar la evolución de los patrones temáticos de los estudiantes durante la resolución de problemas experimentales en el laboratorio es útil para poder tener la información necesaria para prestar a los estudiantes ayuda útil durante esas tareas, si bien otras líneas de investigación, como la relacionada con la modelización (Oliva, 2019), también serían útiles para ello. En concreto, en este estudio nos planteamos analizar la evolución de las relaciones semánticas entre conceptos para dos tareas a las que los estudiantes se enfrentaron en el laboratorio. Estas relaciones se refieren a la conexión de los conceptos con el significado de otros conceptos, y también a su relación con pautas de acción inteligibles y con otros contextos; exploramos en ellas hasta qué punto les resultan funcionales a los estudiantes para implementar pautas de acción útiles para abordar las tareas.

La pregunta de investigación que guía este estudio es: «¿Cómo construyen los estudiantes relaciones semánticas entre conceptos transformándolos en herramientas funcionales para enfrentase a tareas abiertas en el laboratorio?».

\section{CONTEXTO Y METODOLOGÍA}

Los participantes en las dos tareas cuyo análisis mostramos en este estudio fueron un grupo de cuatro estudiantes de $4 .^{\circ}$ de ESO (15-16 años), junto a su profesor (el autor del trabajo), en un instituto público de una ciudad pequeña de España. La clase tenía 22 estudiantes, que fueron divididos en grupos de tres o cuatro. Las tareas analizadas, «Tipo de movimiento en un carril» (TMC) y «Medida de la masa con resortes» (MMR), ocuparon tres sesiones cada una.

Cabe indicar que, en la tarea MMR, hubo algunas modificaciones en los participantes. En la segunda sesión de las dedicadas a ella, tres miembros del grupo no acudieron, cuyo lugar fue ocupado por tres chicas de otro grupo, mientras que en la tercera sesión hubo una fase de intercambio con dos miembros de otro grupo (tabla 1).

Tabla 1.

Participantes en la tarea MMR

\begin{tabular}{|c|c|c|}
\hline Sesión 1 & Sesión 2 & Sesión 3 \\
\hline Susana & Susana & Susana \\
\hline Sergio & Hilaria & Sergio \\
\hline Santiago & Herminia & Santiago/Ramón \\
\hline Simeón & Higinia & Simeón/Ricardo \\
\hline
\end{tabular}

Se procuró que las tareas fueran compatibles con aquellas a las que habitualmente se enfrentan los científicos. Para ello, eran abiertas (en el sentido de tener distintos caminos posibles para llegar a una solución), constituían un problema para los estudiantes, implicaban la necesidad de tomar decisiones metodológicas y epistemológicas y conllevaban también la necesidad de diseñar y llevar a cabo experimentos. Así se pretendía conectar un contexto de ciencia escolar con el de la ciencia real.

En la tarea TMC, los estudiantes debían decidir qué tipo de movimiento sigue una bola moviéndose por un carril en posición horizontal y en posición inclinada. Por su parte, el problema de la tarea MMR consistía en responder a la pregunta de si es posible sustituir una balanza por un resorte a la hora de medir masas, para lo que disponían de dos muelles distintos y una goma. Esta tarea ya había sido puesta anteriormente en práctica con otro grupo de alumnos (Reigosa y Jiménez, 2000). Con ambas tareas se les proporcionaba un guion que establecía el problema que debía resolverse, pero en el que no se les daban instrucciones en forma de pasos concretos. 
El planteamiento de la intervención docente era ayudar a los estudiantes a mejorar la calidad del proceso de resolución de las tareas, no a ayudarles a llegar a toda costa a un resultado determinado. La actividad de los estudiantes fue observada por el profesor, lo que le permitió constatar las dificultades que impedían su progreso en la resolución de la tarea y darles una asistencia orientada a superarlas. La asistencia se concibió en términos de andamiaje, en el sentido de que estaba orientada a ayudar a los estudiantes a progresar en su nivel de competencia autónoma (Mercer y Fischer, 1992). En la interacción profesor-estudiantes se pretendió evitar dar instrucciones de pasos concretos para asegurarse de que habían sido ellas y ellos los que habían dado una solución al problema, lo cual implica necesariamente el desarrollo de las destrezas requeridas para ello.

Los datos analizados en este estudio consisten en las grabaciones de audio y vídeo de los participantes. Esas grabaciones fueron transcritas por el autor y fueron analizadas de forma orientada a la construcción de interpretaciones a partir de los datos, no a apoyar o a refutar hipótesis apriorísticas (Lincoln y Guba, 1985). Para realizar dichas interpretaciones, a la vista de los datos, se optó por una teoría útil para comprenderlos, pareciéndonos adecuada la noción de prácticas contextualizadoras. Las transcripciones fueron revisadas repetidas veces, de manera que se identificaron eventos y regularidades relacionadas con la pregunta de investigación y se formularon interpretaciones que fueron refinadas mediante la revisión continuada y cíclica (McKernan, 1999). El objetivo era llegar a un conocimiento detallado de cómo los diálogos permitieron a los participantes hacer progresar su discurso y sus prácticas.

En el apartado de resultados de este trabajo, se muestran ejemplos que ilustran los aspectos analizados. En ellos, la numeración de las sesiones se corresponde con las dedicadas a cada tarea y la numeración de las intervenciones de los estudiantes, con el total correspondiente a cada sesión. Las manifestaciones orales de los participantes se muestran en letra normal, las acciones físicas en cursiva y los comentarios introducidos por el autor en courier. En los fragmentos de transcripción mostrados se han omitido algunos turnos para reducir la longitud, eliminando aquellos que no contenían informaciones relevantes para los objetivos del trabajo. Algunas intervenciones han sido traducidas del gallego.

\section{RESULTADOS}

\section{Tarea TMC}

Los estudiantes primero abordaron la situación del carril horizontal, y una de las primeras cosas que hacen es medir el tiempo que tarda la bola en recorrer el carril:

Sesión 1 - TMC

\begin{tabular}{|c|c|l|}
\hline 78 & Susana & Dale, venga \\
\hline 83 & Susana & Di ya \\
\hline 92 & Susana & Vale, venga. Vuelve a tirar. Dale \\
\hline 94 & Simeón & $\begin{array}{l}\text { Cuidado que no lo tienes recto eso. Se te va a caer por un lado (refiriéndose a una rampa } \\
\text { pequeña de la que disponían para darle a la bola un impulso ini- } \\
\text { cial para moverla por el carril horizontal) }\end{array}$ \\
\hline 96 & Susana & Luego te digo «ya» cuando llegue aquí, ¿vale? \\
\hline 103 & Sergio & O sea, que esa velocidad... eso es el tiempo, ¿no? Sí. Calcula la velocidad. Es la misma. \\
\hline
\end{tabular}

Aunque Sergio (línea 103) dice que van a calcular la velocidad, después no saben qué hacer con el tiempo que han determinado. 
Sesión 1 - TMC

\begin{tabular}{|l|l|l|}
\hline 112 & Susana & Bien. Entonces y... ¿qué nos dice con este tiempo? \\
\hline 113 & Sergio & El tiempo, pues, para llegar allí. \\
\hline 115 & Susana & Vale. Hay que mirar tipo de movimiento que sigue la bola por el carril, eeeh... pues... \\
\hline
\end{tabular}

En ese momento, no usan los conceptos de tiempo, velocidad y espacio conectados dentro de un patrón temático coherente y útil para resolver la tarea, sino que se trata de conceptos desconectados. Sin embargo, poco después, se produce un importante avance en la resolución de la tarea:

Sesión 1 - TMC

\begin{tabular}{|c|l|l|}
\hline 122 & Susana & Ah, la posición. La posición, pues medimos esto con el rollo este, y que se cumple. \\
\hline 140 & Susana & $\begin{array}{l}\text { Con la fórmula aplicamos... aquí sí sabemos la posición... s }=s_{0}+\mathrm{v} \text { t... entonces sabemos que... que } \\
70 \text { (centímetros ) es igual a } 0+\mathrm{v} \text { x } 1,80 \ldots 1,82 \text { ( segundos }) .\end{array}$ \\
\hline 147 & Simeón & Y esa velocidad la volvemos a calcular abajo. \\
\hline
\end{tabular}

Primero conectan los conceptos a través de una fórmula (línea 140) y después los usan para tomar decisiones (línea 147), siendo capaces de proponer una metodología útil para resolver el problema: medir la velocidad en dos sitios. Así tienen dos valores que pueden comparar para ver si el movimiento es uniforme o no.

Sin embargo, se les plantea una dificultad relacionada con el papel de una rampa pequeña en forma de cuña que les había sido proporcionada con la idea de que la usaran para darle un impulso inicial a la bola cuando esta se moviera por el carril en posición horizontal (figura 1). Interpretamos que identifican movimiento uniforme con la ausencia de una causa que produzca aceleración, lo cual los lleva a la conclusión de que no pueden usar la rampa pequeña (líneas 156-159).

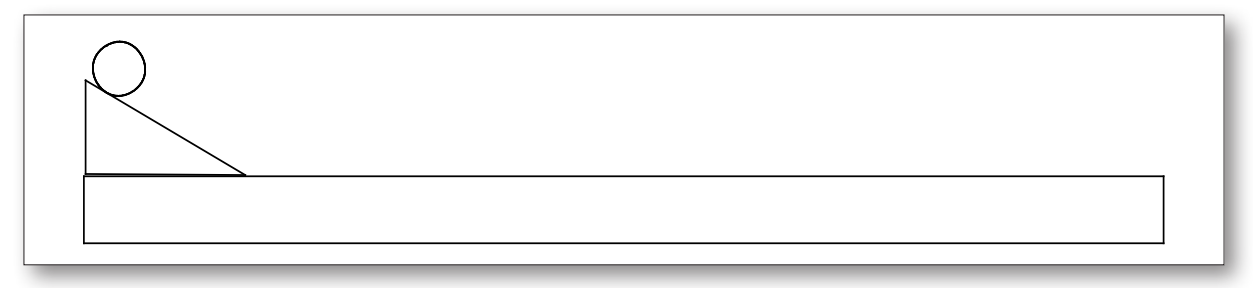

Fig. 1. Movimiento de la bola por el carril horizontal.

\section{Sesión 1-TMC}

\begin{tabular}{|c|l|l|}
\hline 149 & Susana & $\begin{array}{l}\text { Pero es que ¿cómo vamos a saber si es uniformemente acelerado o uniforme simplemente? Yo creo } \\
\text { que es, tiene que ser uniforme. }\end{array}$ \\
\hline 154 & Simeón & Es que no hay nada que lo acelere. \\
\hline 157 & Susana & Sí lo acelera, lo acelera la rampilla \\
\hline 158 & Simeón & $\ldots$ pero entonces no podemos utilizar la rampilla... porque... \\
\hline 159 & Susana & $\begin{array}{l}\text { Claro, es que si es uniforme tiene que ser sin rampa... tiene que ser el recorrido del rel... de, de la bola, } \\
\text { por el carril este, no por este carril... por este carril es, es que acelera, pumba. }\end{array}$ \\
\hline
\end{tabular}


En este momento se encuentran en un callejón sin salida, puesto que, por una parte, son conscientes de que la rampilla afecta al movimiento de la bola, y no saben cómo tener en cuenta ese efecto, y, por otra, también se dan cuenta de que, sin ella, la bola no se mueve. Da la impresión de que no se plantean la posibilidad de estudiar un fenómeno (el movimiento de la bola en el carril horizontal) prescindiendo de su origen (la rampilla). Parecen considerar el movimiento de la bola como un proceso que empieza al dejarla en la rampilla y que no se puede dividir con fines analíticos en etapas. La metodología, habitual en la cultura científica, de estudiar partes de un proceso u objeto por separado es una metodología refinada y poderosa que va más allá del sentido común, como aquí se pone de manifiesto. Vemos que, en este caso, tienen dificultades para plantearse el estudio de movimientos con velocidad inicial distinta de cero, lo cual es imprescindible para lograr una comprensión completa de la primera ley de Newton.

En siguientes fases continúan discutiendo acerca del problema de la rampilla. Proponen eliminarla y empujar la bola, lo cual es desechado por considerar ambas posibilidades equivalentes. En estos momentos, aunque los alumnos hayan desarrollado un contexto indexical con relaciones semánticas correctas entre los conceptos de espacio, tiempo y velocidad, tal contexto no les ha servido para resolver el problema. La existencia de un obstáculo epistemológico originado por la reticencia a estudiar un fenómeno prescindiendo de su origen ha hecho que el marco conceptual no haya sido suficiente. No hacen una conexión entre los aspectos cinemáticos de la tarea (medida de la velocidad) y los dinámicos (los relativos a la fuerza que causa la aceleración requerida para iniciar el movimiento). Un contexto indexical consta de más dimensiones que las relaciones temáticas entre los conceptos, los cuales se pone de manifiesto que no son lo único que hay que tener en cuenta para que un contexto indexical sea operativo. Tras un intervalo de tiempo en el que no son capaces de proseguir consultan con el profesor:

Sesión 1 - TMC

\begin{tabular}{|l|l|l|}
\hline 282 & Susana & Pero se supone que si estuviera horizontal-horizontal tendría que estar quieta la bola. \\
\hline 283 & Profesor & Ya, pero... \\
\hline 285 & Profesor & cuando se mueve. \\
\hline 286 & Susana & ¿Dándole una velocidad inicial? \\
\hline 287 & Profesor & Sí. \\
\hline 291 & Susana & $\begin{array}{l}\text { Ya, pero me refiero si ponemos la rampa, entonces la, la, le estamos dando la velocidad, o sea, es } \\
\text { distinta... no estamos utilizando la longitud del carril. Sin embargo, si le doy... un golpe desde aquí. }\end{array}$ \\
\hline 292 & Profesor & $\begin{array}{l}\text { Bueno, pero se refiere a cuando está circulando por el carril. Entonces tú no contarías desde el prin- } \\
\text { cipio de la rampa. }\end{array}$ \\
\hline 293 & Sergio & No contaría desde el principio. \\
\hline
\end{tabular}

La solución aportada por el profesor es trivial desde el punto de vista experto: estudiar el movimiento solo donde no está la rampilla inicial. Sin embargo, para los alumnos esto supuso un obstáculo que no fueron capaces de superar.

Una vez recibida esa asistencia por parte del profesor, son capaces de controlar las condiciones iniciales:

Sesión 1 - TMC

\begin{tabular}{|l|l|l|l|}
\hline 305 & Susana & Hay que elegir una posición. \\
\hline 306 & Sergio & Es que hay que hallar la velocidad... Es que, espera, la velocidad es ese, te... \\
\hline 312 & Simeón & $\begin{array}{l}\text { El tiempo, el tiempo lo tenemos que calcular desde que sale de aquí (donde se acaba la } \\
\text { rampilla ...., no desde arriba, con la, con la... }\end{array}$ \\
\hline
\end{tabular}


Un poco más adelante dan con una forma de resolver la tarea:

Sesión 1 - TMC

\begin{tabular}{|c|l|l|}
\hline 533 & Susana & Entonces, ahora, hallamos la, la, ¿cómo se llama? Mm... la velocidad cuando la posición es, eeeh... \\
\hline 537 & Susana & $\begin{array}{l}\text {... cuando la posición es cero seiscientos diecinueve (metros ). Si hallamos, si cogemos otra posi- } \\
\text { ción, otro punto, si hallamos la velocidad, y, si, la, la velocidad coincide, es que... }\end{array}$ \\
\hline 539 & Susana & ... es uniforme, y si no, es que es acelerado, o decelerado, claro. ¿Qué, qué posición cogemos? \\
\hline
\end{tabular}

Aquí son capaces de usar los conceptos de forma satisfactoria como herramientas para llevar a cabo la toma de decisiones que permitan resolver el problema. Aquí ya no persiste el obstáculo epistemológico anterior y el contexto indexical activado permite insertar en él las acciones necesarias para resolver la tarea. Vemos la importancia de considerar aspectos tales como la existencia de obstáculos epistemológicos como, en este caso, las dificultades para estudiar un fenómeno separadamente de su origen. La reconstrucción hecha en este trabajo de las fases de esta tarea y que van desde los conceptos inicialmente desconectados hasta los conceptos como herramientas útiles para tomar decisiones se muestra en la figura 2 .

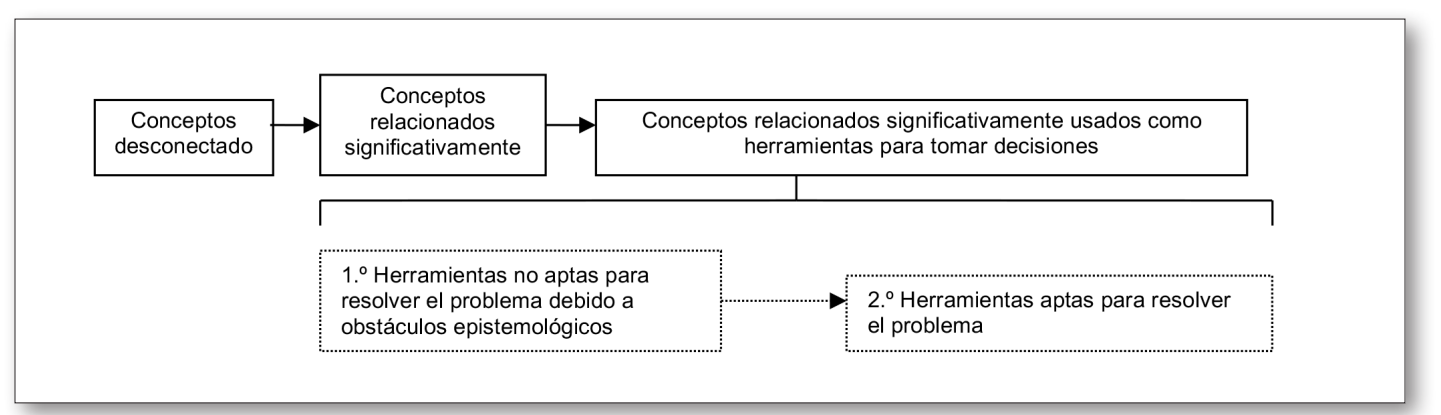

Fig. 2. Fases en la construcción de los aspectos temáticos del contexto indexical (carril horizontal).

Después de enfrentarse al problema del carril horizontal pasan a analizar la situación de cuando este está inclinado. Hacen la planificación de las acciones a ejecutar con mucha mayor rapidez, debido a que se basan en el contexto indexical desplegado durante la primera parte de la tarea, en el cual insertan satisfactoriamente acciones útiles para resolver el problema. Miden para dos tramos de distinta longitud, y obtienen los resultados de la tabla 2.

Tabla 2.

Datos obtenidos inicialmente por los alumnos para el carril inclinado

\begin{tabular}{|c|c|c|}
\hline $\mathrm{s} / \mathrm{m}$ & $\mathrm{t} / \mathrm{s}$ & $\mathrm{v}_{\mathrm{m}} /\left(\mathrm{m} \mathrm{s}^{-1}\right)$ \\
\hline 0,619 & 1,10 & 0,56 \\
\hline 0,400 & 0,75 & 0,53 \\
\hline
\end{tabular}

Estos resultados no conducen a la conclusión de que es un movimiento uniformemente acelerado y la semejanza de las velocidades les hace pensar que entre ellas no hay diferencias significativas: 
Sesión 2 - TMC

\begin{tabular}{|r|l|l|}
\hline 84 & Susana & ¿Cómo va a ser uniforme si va... aumentando? \\
\hline 100 & Susana & Mira, hasta que nos dé un resultado distinto, va mal. \\
\hline
\end{tabular}

Puede pensarse que Susana se niega a aceptar los datos experimentales, pero hay que tener en cuenta que los resultados que obtienen son anómalos. Al hacer las medidas, obtienen el resultado anómalo de que la velocidad es igual para los dos tramos, lo cual no es coherente con lo que observan. La alumna es capaz de usar el contexto indexical que maneja para generar un criterio y poner así en duda la validez de determinados datos experimentales.

En ocasiones se ha señalado la resistencia de los estudiantes a variar sus esquemas conceptuales frente a situaciones empíricas que están en conflicto con ellas (ver, por ejemplo, Driver, Guesne y Tiberghien, 1989), pero centrándose en casos en los que se buscaba que los estudiantes abandonasen esquemas erróneos desde el punto de vista de la ciencia. Sin embargo, en el caso que aquí mostramos, los estudiantes están en lo cierto, puesto que es correcto que la velocidad no es constante, sino que va aumentando. Como muestran Chinn y Brewer (1993), la modificación de las teorías solo es una de las múltiples posibilidades que se abren ante unos datos u observaciones anómalos con respecto a un marco de referencia. De hecho, la identificación de datos anómalos no es una destreza universal y desligada del aprendizaje y del contexto (Toplis, 2007), por lo que se han planteado proyectos educativos que incluyen ayudar a los alumnos a identificar datos sospechosos (Wood-Robinson et al., 2000). Más adelante, cuando hablan con el profesor aclaran por qué el resultado es anómalo, haciendo una referencia a otro contexto (la bicicleta):

Sesión 2 - TMC

\begin{tabular}{|c|l|l|}
\hline 425 & Profesor & ¿Por qué dices... por qué no puede ser uniforme cuando está inclinado? \\
\hline 429 & Susana & $\begin{array}{l}\text { Si vas en bicicleta, cuando te pillas una cuesta mm, y dejas, bueno, pues en punto muerto, pues vas } \\
\text { aumentando. }\end{array}$ \\
\hline 433 & Sergio & Si te dejas caer por una rampa, al llegar al fondo vas con mucha más velocidad que arriba. \\
\hline
\end{tabular}

Poco tiempo después, el profesor les ayuda mostrándoles que el error está en la excesiva inclinación con que habían colocado el carril:

Sesión 2 - TMC

\begin{tabular}{|l|l|l|}
\hline 536 & Profesor & $\begin{array}{l}\text { Cuando va muy rápido, ¿sabéis qué pasa? Es muy difícil medir bien los tiempos. Entonces, vamos a } \\
\text { hacerlo así... }\end{array}$ \\
\hline 537 & Profesor & (Coloca el carril con mucha menos inclinación) \\
\hline 543 & Profesor & ¿Entendéis? Si lo hacemos todo muy rápido, es muy difícil medir los tiempos. \\
\hline
\end{tabular}

El conocimiento manejado por el profesor incluye aspectos procedimentales vitales como mayor destreza acerca del control del error experimental. El profesor intenta que se den cuenta de que, como las magnitudes físicas pequeñas son difíciles de medir con exactitud, es muy inexacto medir tiempos pequeños. Por ello, lo que corresponde hacer es inclinar menos el carril para obtener tiempos mayores.

Después, hacen las mediciones con una inclinación menor para los dos tramos, introducen el concepto de aceleración y resuelven el problema. En resumen, en la segunda parte de la tarea TMC, se vuelve a poner de manifiesto que un contexto indexical en el que los conceptos simplemente son valorados en términos de comprensión de sus relaciones semánticas no resulta suficiente, sino que aquí 
interfieren dificultades de tipo procedimental. Las fases en la adecuación de las relaciones semánticas entre conceptos del contexto indexical para la resolución de la segunda parte de este problema se muestran en la figura 3:

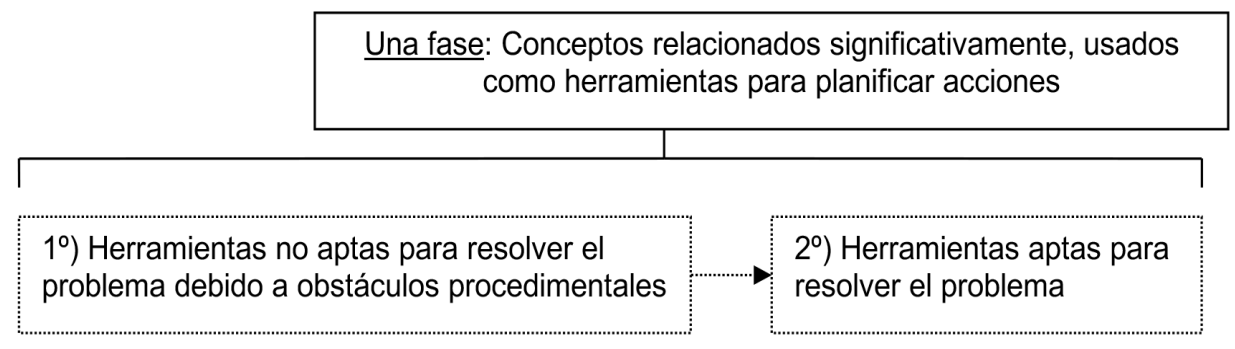

Fig. 3. Fases en la construcción de los aspectos temáticos del contexto indexical (carril inclinado).

Resulta evidente que en la segunda parte de esta tarea los estudiantes se beneficiaron del contexto indexical evocado en la primera parte. Se produjo, pues, una transferencia de conocimientos desde la primera a la segunda parte.

\section{Tarea MMR}

En esta tarea se les suministraban a los estudiantes diversos resortes (dos muelles y una goma) con el fin de que decidieran cuál era el más adecuado para ser usado en sustitución de una balanza para medir masas. Los estudiantes enseguida hacen propuestas de acciones para llevar a cabo:

\section{Sesión 1 - MMR}

\begin{tabular}{|c|l|l|}
\hline 117 & Santiago & $\begin{array}{l}\text { A ver, pues yo pienso que, más que nada, antes debíamos colocar el rollo (el montaje) para } \\
\text { poder hacer la práctica. }\end{array}$ \\
\hline 128 & Sergio & ¿Qué tenemos que hacer? Hacer, medir con un muelle. \\
\hline 129 & Susana & $\begin{array}{l}\text { Sí, se supone. Medir el muelle sin peso, y medirlo con el peso, y la diferencia entre los dos se halla } \\
\text { (estirando un muelle) }\end{array}$ \\
\hline 130 & Sergio & Aaah. Tenemos que llevarlo a la balanza. \\
\hline
\end{tabular}

Los estudiantes relacionan el peso colgado del resorte con el estiramiento de este. Relacionan los conceptos de peso y estiramiento y, además, los usan como herramientas para la toma de decisiones. Sin embargo, las propuestas que realizan carecen de utilidad puesto que no están relacionadas con el objetivo general de la tarea. Es decir, proponen acciones sin saber para qué sirven. Al poco tiempo, los estudiantes recuerdan la ley de Hooke:

\section{Sesión 1 - MMR}

\begin{tabular}{|l|l|l|}
\hline 257 & Santiago & ¿No había una fórmula para esto? \\
\hline 270 & Susana & Era $l-1_{0} \ldots$ \\
\hline 272 & Susana & y por $k$, igual a F... que es la fuerza. \\
\hline
\end{tabular}


Parece que ahora los estudiantes relacionan los conceptos de una forma más compleja que antes, cuando simplemente eran conscientes de que los pesos colgados del resorte causan un estiramiento. En estos momentos, los estudiantes relacionan estiramiento $(l-l)$ con peso $(F)$ mediante la expresión matemática de la ley de Hooke. Esa ley implica ir más allá de lo hecho hasta ese momento, que era simplemente apuntar implícitamente la existencia de una relación entre peso y estiramiento, ya que la ley de Hooke implica además la especificación de un tipo concreto de relación, una relación lineal. Sin embargo, al poco tiempo se pone de manifiesto que la comprensión por parte de los estudiantes de esa relación es incompleta, ya que ni siquiera tienen claro el significado de los parámetros que aparecen en la ley de Hooke.

\section{Sesión 1 - MMR}

\begin{tabular}{|l|l|l|}
\hline 405 & Susana & ¿La $k$ era el peso? \\
\hline 406 & Sergio & Claro, para hallar la fuerza. Sí. \\
\hline 412 & Santiago & Once por siete. La fuerza es once por siete. \\
\hline 413 & Susana & Ya, eso ya lo sé, pero me refiero a que... $k$ es eso (señalando el portapesas). \\
\hline 417 & Sergio & Sí. $k$ es el peso, profe, ¿`no? $k$ es el... \\
\hline 419 & Profesor & F es el peso, que es la fuerza. \\
\hline 420 & Susana & ¿Y entonces $k$ qué era? \\
\hline 421 & Profesor & $k$ es una constante de cada muelle. \\
\hline 429 & Profesor & Constante elástica. \\
\hline 431 & Santiago & ¿Pero, pero cuánto vale? \\
\hline 433 & Profesor & Pues depende, depende. \\
\hline 437 & Susana & ¿Y había una fórmula para eso? ¿Para hallarla? \\
\hline 439 & Profesor & Solo hay esa fórmula. \\
\hline
\end{tabular}

El profesor aclara qué representan F y $k$ en la fórmula, y se pone de manifiesto el bajo dominio que tenían los estudiantes de la ley de Hooke. La ley de Hooke hasta estos momentos no suponía para los estudiantes más que una relación formal de naturaleza memorística entre conceptos, no una relación significativa, a pesar de que ellos comprenden perfectamente que, a mayor peso, mayor estiramiento. Parece que el problema es que no saben cómo la ley de Hooke representa esa relación.

A continuación, prosiguen discutiendo acerca de la ley de Hooke, preocupándose por las unidades de las magnitudes físicas implicadas:

\section{Sesión 1 - MMR}

\begin{tabular}{|l|l|l|}
\hline 463 & Susana & Hay que... ¿y qué dije? ¿Y en qué unidades está? \\
\hline 471 & Santiago & En gramos. \\
\hline 477 & Susana & ¿Y entonces por qué se pasaba a metros aquella cosa? \\
\hline 485 & Sergio & Un Newton. Se mide en Newton (leyendo en la libreta). \\
\hline
\end{tabular}

Su interés en estos momentos se centra en aclarar aspectos formales, como las unidades de las magnitudes físicas implicadas, sin ser capaces de tomar decisiones útiles de cara al objetivo general de la tarea. Pero, poco después, hacen una propuesta de acción basada en la ley de Hooke:

\section{Sesión 1 - MMR}

\begin{tabular}{|l|l|l|}
\hline 533 & Sergio & Tenemos que hallar la, la constante de cada muelle. \\
\hline 543 & Susana & Es que... no sabemos hallar la constante de cada muelle. \\
\hline
\end{tabular}


Los alumnos han pasado a una situación de manejo de conceptos relacionados, usándolos además como herramientas para planificar acciones. En la manifestación de la línea 533 se da por supuesto que cada resorte tiene una constante elástica, es decir, que la ley de Hooke es cumplida por cualquier resorte. Además, los estudiantes están tomando como objetivo de la tarea reunir valores para sustituirlos en la ley de Hooke, sin relacionar eso con el objetivo general de la tarea. Dar por sentado que todo resorte cumple la ley de Hooke implica no considerar la posibilidad de distintas relaciones entre peso y estiramiento, lo cual es importante para abordar el problema. Las relaciones entre peso y estiramiento podrían ser lineales (ley de Hooke) o de otros tipos y habría que pensar cuál es la más conveniente, pero los estudiantes en ningún momento se han planteado la posibilidad de la existencia de distintas relaciones, lo cual puede deberse a no considerar que haya distintos tipos de relaciones entre magnitudes. No consideran otras posibilidades distintas del modelo lineal. Eso constituye un obstáculo procedimental que limita el logro del objetivo planteado.

En los momentos siguientes, hacen las medidas necesarias para calcular el valor de la constante elástica, tras lo cual se producen nuevas propuestas de acciones que ejecutar:

\section{Sesión 1 - MMR}

\begin{tabular}{|l|l|l|}
\hline 871 & Sergio & Pues ahora tenemos que hacer lo mismo con todos los objetos. \\
\hline 872 & Susana & Vale, ¿y esto qué? ¿Qué nos da? \\
\hline
\end{tabular}

Sergio propone determinar la constante elástica con otros resortes, pero su propuesta carece de conexión explícita o compartida por el grupo con relación al objetivo general de la tarea. No analizan por qué usar la ley de Hooke podría resultar útil a la vista del objetivo general de la tarea, sino que su mero uso les parece ya de por sí satisfactorio. Parece darse por supuesto que del mero uso de la fórmula surgirá espontáneamente la resolución del problema. La consideración de las fórmulas como panacea universal en la resolución de problemas puede verse reforzada por la resolución de ejercicios en el aula que se reducen a la sustitución de datos en la fórmula adecuada, lo cual no es infrecuente en la enseñanza tradicional de la física en el nivel secundario. Esos ejercicios pueden conducir al desarrollo de un contexto indexical estereotipado en el cual se siguen rutinas a pesar de no estar relacionadas con la construcción de conocimiento. Sin embargo, es relevante la manifestación de Susana de la línea 872, en la que se busca romper esa dinámica al preguntar qué obtienen con su forma de proceder.

En la siguiente sesión, se siguen poniendo de manifiesto importantes lagunas en la comprensión de la ley de Hooke:

\section{Sesión 2 - MMR}

\begin{tabular}{|l|l|l|}
\hline 177 & Profesor & ¿Exactamente para que medís $k$ ? \\
\hline 188 & Susana & ¿Cómo? Pues cuando supiéramos $k$ podríamos despejar F, despejar esta fórmula. \\
\hline 189 & Profesor & Y eso os vale para saber cuánto es el peso... \\
\hline 190 & Profesor & Bueno, pues podéis comprobar si se puede hacer eso. \\
\hline 192 & Susana & Ya, ya, ya lo la ( inaudible). Da unos valores, da unos valores de $k$ muy distintos. \\
\hline 201 & Profesor & No tiene por qué dar lo mismo, eh, cada muelle. \\
\hline 208 & Profesor & $\begin{array}{l}\text { Pero hay que tener en cuenta una cosa. La ley de Hooke no la cumple cualquier muelle o goma, eh. } \\
\text { Solo la cumplen algunos }\end{array}$ \\
\hline 210 & Profesor & (inaudible) si no se cumple... \\
\hline 212 & Profesor & no podéis aplicarla después \\
\hline
\end{tabular}


El profesor aclara que el valor de la constante elástica es distinto para cada resorte, ya que Susana había manifestado su extrañeza por haber obtenido valores distintos. Además, el profesor tiene que aclarar que la ley de Hooke no es de cumplimiento universal. La falta de comprensión de la posibilidad de la existencia de distintas relaciones que conectan peso y estiramiento constituye un obstáculo para que los estudiantes puedan ser capaces de resolver el problema.

No es hasta la siguiente sesión cuando toman decisiones basadas en el contexto indexical construido según esa relación.

\section{Sesión 3 - MMR}

\begin{tabular}{|c|l|l|}
\hline 65 & Susana & $\begin{array}{l}\text { Yaaa, pero es que ahora hay que hacerlo con dos pesos distintos, y la constante que sea más constante } \\
\text { que... es el muelle que, que hay que usar }\end{array}$ \\
\hline
\end{tabular}

En estos momentos ya son capaces de usar sus conocimientos para tomar decisiones de utilidad para abordar el problema, ya que las acciones que ponen en juego permiten comprobar qué resorte cumple mejor la ley de Hooke. Pero consideramos sus decisiones parcialmente útiles porque consideran acríticamente que el buen cumplimiento de la ley de Hooke es sinónimo de que un resorte sirva para determinar masas, sin considerar la posibilidad de otras relaciones peso-estiramiento y sin aclarar por qué una relación lineal es preferible a otras. No plantearse la posible existencia de distintas relaciones constituye un obstáculo procedimental que los estudiantes no han sido capaces de superar y que ha limitado sus logros. En la figura 4 se muestra su avance en términos de construcción de relaciones semánticas entre conceptos.

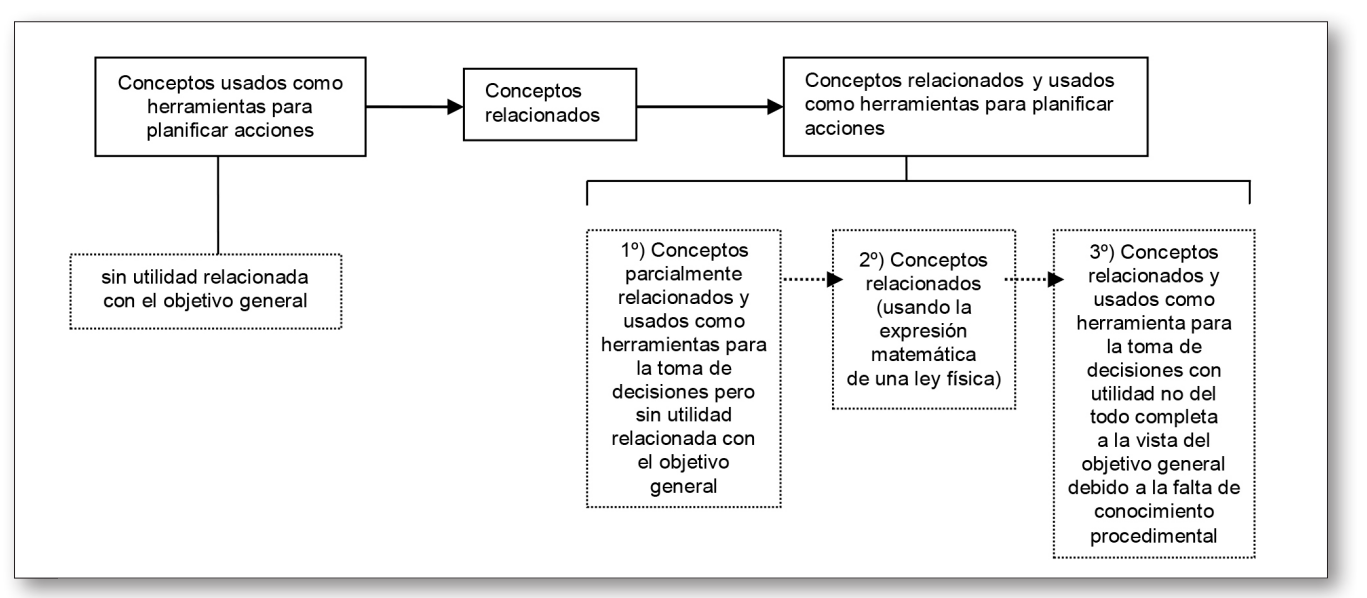

Fig. 4. Fases en la construcción de los aspectos temáticos del contexto indexical (tarea MMR).

Más adelante se produce una fase de intercambio de Susana y Sergio con Ramón y Ricardo, de otro grupo, en la que se ponen de manifiesto las carencias de sus logros. Durante esta fase, el profesor les había pedido que comparasen sus resultados:

Sesión 3 - MMR

\begin{tabular}{|l|l|l|}
\hline 545 & Ricardo & A ver, venga, la goma es la más exacta. \\
\hline 548 & Sergio & ¿Por? \\
\hline 550 & Sergio & Porque hay que hallar los cálculos esos. \\
\hline 551 & Ramón & ( La goma ) es la más elástica, ¿̇no ves? \\
\hline
\end{tabular}


La justificación que da Ramón (línea 551) de que la goma es la más elástica puede parecer improcedente, pero no lo es, ya que tiene su motivación, puesto que su grupo no usa explícitamente la ley de Hooke, sino que estudia la proporcionalidad estiramiento-peso mediante reglas de tres, y la medida del estiramiento es más fácil cuanto más deformable sea el muelle (lo que Ramón denomina «elasticidad»). Esta situación es un ejemplo, además, de conexión entre contextos.

Después, Ramón les explica el fundamento de su método, en el que obtienen la masa de un objeto con un resorte en base a la aplicación de una regla de tres y después comparan con su masa medida en una balanza. Pensamos que cabe considerar más fundamentada la metodología del grupo de Ricardo y Ramón que la del grupo de Susana y Sergio, que valoran el cumplimiento de la ley de Hooke sin más.

Sesión 3 - MMR

\begin{tabular}{|l|l|l|}
\hline 565 & Sergio & Eh, ¿̇uál es la diferencia entre una constante y la otra? \\
\hline 568 & Ramón & Es que nosotros no hicimos así. \\
\hline 573 & Ramón & Primero fuimos mirando qué... \\
\hline 577 & Ramón & cómo estiraba cada cosa con cierto peso... \\
\hline 579 & Ramón & Esto después le pusimos... eeh, otro peso, más grande, por ejemplo, y le hicimos una regla de tres... \\
\hline 581 & Ramón & con cada uno, y lo que sea más exacto, pesamos en la balanza... y nos daba esto. \\
\hline 582 & Susana & Yos da la goma. \\
\hline 583 & Ramón & Sí. ¿ Y vosotros? \\
\hline 597 & Sergio & Pero nosotros llegamos a la conclusión de que era esto, no sé por qué, pero bueno. \\
\hline
\end{tabular}

Sergio manifiesta que no sabe el porqué de su conclusión y que usar la fórmula es más exacto (línea 550). Parece que piensa que considerar el uso de fórmulas es per se una justificación, es decir, que el uso de fórmulas goza de un estatus superior al de otros métodos. Podemos decir que el uso de la ley de Hooke aquí inhibe el razonamiento más que promoverlo, puesto que los estudiantes se conforman con usarla, pero sin aclarar por qué.

En definitiva, los alumnos son capaces de desarrollar un contexto indexical que les permite dar los pasos necesarios para ver qué resorte cumple mejor la ley de Hooke. Sin embargo, la falta de conocimiento procedimental relativo a que puede haber distintos tipos de relaciones entre variables limita sus logros, no siendo capaces de resolver el problema de forma satisfactoria al quedar en el aire por qué el cumplimiento de la ley de Hooke hace que un resorte sea mejor para medir masas que otros que no la cumplen.

\section{REGULARIDADES EN LA CONSTRUCCIÓN DE RELACIONES SEMÁNTICAS}

En la tarea TMC parten de conceptos desconectados, conectándolos después y usándolos más tarde como herramientas para tomar decisiones, si bien inicialmente esas herramientas carecían de funcionalidad debido a obstáculos epistemológicos. Después sí son capaces de resolver la tarea satisfactoriamente. Cuando pasan a la parte de la tarea en la que se analiza el movimiento en un carril inclinado, usan los conceptos como herramientas para tomar decisiones, pero aquí también hay dificultades iniciales, en este caso procedimentales, que son superadas, como antes, con la ayuda del profesor.

En la tarea MMR, ya desde el principio usan los conceptos relevantes relacionados como contexto indexical de cara a la toma de decisiones, aunque esas decisiones no tengan utilidad de cara al objetivo general de la tarea. Después se producen cambios en su forma de ver la relación entre conceptos, aunque no llegan a ser capaces de usarlos como herramientas con la funcionalidad necesaria como 
para tomar decisiones con utilidad completa a la vista del objetivo general de la tarea. Consideramos esto debido a la falta de conocimiento acerca de que puede haber distintos tipos de relaciones entre variables, lo cual constituye un obstáculo procedimental. Después, optan por sustituir valores en una fórmula sin quedar claro por qué eso es adecuado.

Viendo conjuntamente lo sucedido en las tareas, consideramos que hay dos dimensiones parte del contexto indexical necesario para resolver el problema y que se deben considerar para valorar las dificultades de los estudiantes a la hora usar las relaciones semánticas entre conceptos construidas:

- La importancia de la existencia de obstáculos epistemológicos o procedimentales.

- La importancia para los estudiantes de la complejidad de las relaciones semánticas implicadas.

En las tareas analizadas se ponen de manifiesto obstáculos epistemológicos y procedimentales que limitan los logros de los alumnos. Se pone de manifiesto que, en lo que se refiere a los conceptos, no importa solo conocer su significado, sino también saber cómo se usan, incluyendo los conocimientos prácticos que su aplicación implica. Esto se pone de manifiesto con esas dos tareas. En lo que a esto respecta, el papel del profesor es de gran relevancia, por ejemplo, en la tarea TMC, en la que las dificultades procedimentales y epistemológicas son superadas con ayuda del profesor, quien pudo constatarlas observando el desempeño de los miembros del grupo.

En resumen, para que un contexto indexical tenga la funcionalidad necesaria para resolver un problema, no solo son importantes los patrones temáticos, es decir, las relaciones semánticas entre conceptos, sino que, en este estudio, se han puesto de manifiesto más dimensiones: dos son el conocimiento procedimental y los compromisos epistemológicos. El conocimiento procedimental es el dominio de técnicas y metodologías, incluyendo el manejo del instrumental relevante. En cuanto a los compromisos epistemológicos, siguiendo a Sandoval y Morrison (2003), entendemos como tales las teorías implícitas acerca de cómo el conocimiento científico es construido, qué es el trabajo científico o el propósito y métodos del trabajo experimental.

Además, consideramos que hay otra dimensión que se pone de manifiesto cuando, en la tarea MMR, los estudiantes optan por sustituir datos en la ley de Hooke sin tener clara su utilidad, posiblemente por considerar que sustituir valores en fórmulas es una metodología que se ha de seguir siempre que se pueda. Esa idea es una rutina o norma implícita que también les sirve para tomar decisiones y dar pasos, y por tanto también forma parte del contexto indexical.

Las distintas dimensiones que hemos identificado para los contextos indexicales se muestran en la figura 5, la cual se refinó y enriqueció en interacción con los datos, y basándonos en las ideas expuestas en el marco teórico de este trabajo.

En cuanto a la complejidad, desde el punto de vista de los estudiantes, de las relaciones semánticas entre los conceptos relevantes, se ha constatado su importancia y su influencia, ya que tareas con distinto grado de dificultad conducen a situaciones diferentes. Se ha puesto de manifiesto la necesidad de relacionar conceptos, o de refinar dichas relaciones. Por ejemplo, en la tarea MMR, en la que introducen, al cabo de un cierto tiempo, la ley de Hooke para relacionar peso y estiramiento. En unas tareas la introducción de los conceptos relevantes se hace de manera más rápida que en otras, pero en general se ha observado que es necesario que los estudiantes tengan oportunidades para construir las relaciones necesarias entre ellos, lo cual es un estadio previo a usarlos como herramientas para la toma de decisiones. Consideramos que se puede decir que la evolución de los patrones temáticos no es simplemente un proceso cuantitativo por el que ańadir más conceptos o aumentar o refinar las relaciones entre estos, sino que, además, es un proceso cualitativo en el cual las relaciones semánticas entre conceptos atraviesan transformaciones de su funcionalidad. En estas transformaciones se atraviesan fases que no son las mismas en distintas tareas. 


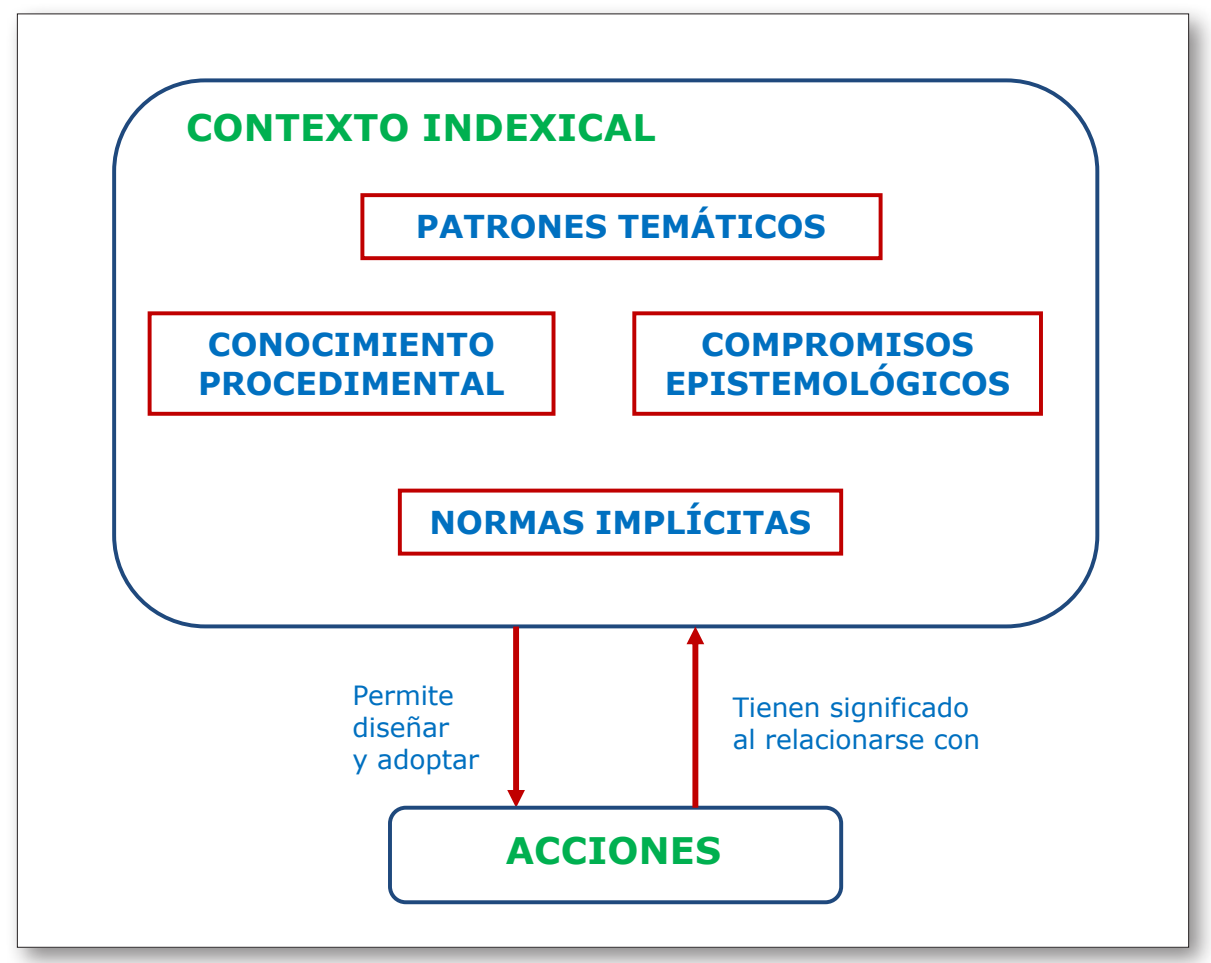

Fig. 5. Componentes identificados en los contextos indexicales.

\section{CONCLUSIONES}

En este trabajo se ha analizado el proceso que siguen grupos de estudiantes para construir un patrón temático funcional para enfrentarse en el laboratorio de física a tareas concebidas como problemas. Los patrones temáticos, o conjuntos de relaciones semánticas entre conceptos (Lemke, 1997), son fundamentales para que los estudiantes sean capaces de resolver un problema: solo si los estudiantes disponen de un patrón temático adecuado, lo cual implica que sea coherente y aceptable desde el punto de vista disciplinar, pueden enfrentarse con éxito al problema.

Hemos visto en los resultados de este estudio que los patrones temáticos correspondientes a cada tarea son desarrollados por parte de los estudiantes mediante un proceso a veces laborioso. Las acciones son significativas solamente si se colocan en un contexto, y aquí los estudiantes no disponen desde el principio de tal contexto, por no haber refinado suficientemente las imprescindibles relaciones entre conceptos. De ahí que inicialmente no sean capaces de conectar las acciones con los conceptos que les darían significado y no sean capaces de resolver los problemas.

En el estudio se ha encontrado que el desarrollo de patrones temáticos adecuados para resolver el problema es un proceso en el que las relaciones semánticas entre conceptos sufren una transformación cualitativa que atraviesa distintas fases cuya naturaleza depende de cada tarea y de la capacidad de activar contextos indexicales adecuados. Unas veces, el significado que tienen inicialmente los conceptos es el de simples definiciones, las cuales van cobrando progresivamente significatividad. Además, puede suceder que algunos conceptos que hay que usar significativamente de forma integrada para enfrentarse a la tarea no estén relacionados inicialmente y los estudiantes tengan que hacer frente a su conexión. 
Es relevante que puedan darse casos en los que planifiquen acciones sin que las relaciones relevantes entre conceptos sean significativas, es decir, sin contar con un contexto indexical aceptable desde el punto de vista científico. Un ejemplo es la tarea MMR, en la que hay fases en las que se dan pasos sin conocer su utilidad simplemente para simular un rol de alumno activo y correcto porque sustituye valores en fórmulas. Cumplir con normas o costumbres implícitas (que no siempre son útiles) también es parte del contexto indexical. Esto es coherente con los resultados de Adler et al. (2018), que muestran la influencia del contexto escolar en la calidad de los procesos de indagación abierta del alumnado.

Además, se han observado situaciones en las que, aunque los estudiantes comprendan las relaciones más relevantes entre los conceptos implicados, pueden existir obstáculos tanto de tipo epistemológico como procedimental que limiten su grado de avance. Como Kelly y Cunningham (2019) muestran, sin los recursos epistémicos necesarios los estudiantes no pueden enfrentarse con éxito a determinadas tareas.

Como implicaciones educativas señalamos que, al enfrentarse los estudiantes en el laboratorio a una tarea práctica concebida como la resolución activa de un problema, es conveniente, con el fin de prever las dificultades que van a experimentar y el grado de asistencia que van a necesitar, analizar la dificultad que tiene el patrón temático relevante, así como los contextos disponibles para el alumnado y la posibilidad de la existencia de obstáculos de diferentes tipos.

De todas formas, aunque valorar los posibles obstáculos sea útil, estamos convencidos de que hay ocasiones en las que van a surgir algunos inesperados, que actuarán como factores que limiten el grado de avance de los estudiantes. A este respecto, se hace necesaria la observación atenta por parte del profesor de la actividad de los estudiantes durante el desarrollo de las tareas, para así constatar las dificultades que experimentan y poder prestarles asistencia concebida como andamiaje, ayudándolos a completar las tareas y, sobre todo, a alcanzar un mayor nivel de competencia autónoma y transfiriendo una responsabilidad creciente a los estudiantes para que puedan evocar contextos indexicales útiles. Como Kim y Pegg (2019) señalan, el apoyo del profesor es de especial importancia en tareas concebidas en términos de resolución de problemas, y, además, el análisis de la actuación de los profesores puede contribuir a acercar las innovaciones e investigaciones educativas a la práctica docente (Contreras, 2017). Realizar una misma tarea con los estudiantes en cursos sucesivos, además, puede proporcionar una rica fuente empírica de conocimiento acerca de las dificultades que los estudiantes experimentan más habitualmente.

\section{REFERENCIAS BIBLIOGRÁFICAS}

Adler, I., Schwartz, L., Majdar, N. y Zion, M. (2018). Reading between the lines: the effect of contextual factor on students motivation throughout an open inquiry process. Science Education, 102, 820-855. https://doi.org/10.1002/sce.21445

Barab, S. A. y Hay, K. E. (2001). Doing science at the elbows of experts: issues related to the science apprenticeship camp. Journal of Research in Science Teaching, 38(1), 70-102. https://doi.org/10.1002/1098-2736(200101)38:1<70::AID-TEA5>3.0.CO;2-L

Bell, R. L., Blair, L. M, Crawford, B. A. y Lederman, N. G. (2003). Just do it? Impact of a science apprenticeship program on high school students' understandings of the nature of science and scientific inquiry. Journal of Research in Science Teaching, 40(5), 487-509.

https://doi.org/10.1002/tea.10086 
Bjørkvold, T y Blikstad-Balas, M. (2018). Students as researchers: what and why seventh-grade students choose to write when investigating their own research question. Science Education, 102, 304-341. https://doi.org/10.1002/sce.21324

Brown, J. S., Collins, A. y Duguid, P. (1989). Situated cognition and the culture of learning. Educational Researcher, 18(1), 32-42. https://doi.org/10.3102/0013189X018001032

Cazden, C. B. (1988). Classroom discourse. The language of teaching and learning. Londres: Routledge $\&$ Kegan Paul.

Charney, J., Hmelo-Silver, C. E., Sofer, W., Neigeborn, L., Coletta, S. y Nemeroff, M. (2007). Cognitive apprenticeship in science through immersion in laboratory practices. International Journal of Science Education, 29(2), 195-213. https://doi.org/10.1080/095006906005609585

Chinn, C. A. y Brewer, W. F. (1993). The role of anomalous data in knowledge acquisition: a theoretical framework and implications for science instruction. Review of Educational Research, 63(1), $1-49$. https://doi.org/10.2102/00346543063001001

Contreras, S. (2017). Análisis del pensamiento y la actuación del profesor de ciencias. Eje de desarrollo para acercar la investigación a la práctica e innovar en la formación inicial de profesores. Enseñanza de las Ciencias. Número especial X Congreso Internacional sobre Investigación y Enseñanza de las Ciencias, 81-87.

Driver, R., Guesne, E. y Tiberghien, A. (1985). Children's ideas in science. Buckingham: Open University Press.

Godino, J. D., Gonzato, M., Cajaraville, J. A. y Fernández, T. (2012). Una aproximación ontosemiótica a la visualización en educación matemática. Enseñanza de las Ciencias, 30(2), 119-130. https://doi.org/10.5565/rev/ec/v30n2.653

Kelly, G. J. y Cunningham, C. M. (2019). Epistemic tools in engineering design for K-12 education. Science Education, 103, 1080-1111.

https://doi.org/10.1002/sce.21513

Kim, M. y Pegg, J. (2019). Case analysis of children's reasoning in problem-solving process. International Journal of Science Education, 41(6), 739-758. https://doi.org/10.1080/09500693.2019.1579391

Kuhn, D., Sin Arvidsson, T., Lesperance, R. y Corprew, R. (2017). Can engaging in science practices promote deep understanding of them? Science Education, 101(2), 232-250.

https://doi.org/10.1002/sce.21263

Lemke, J. (1997). Aprender a hablar ciencia. Barcelona: Paidós.

Lincoln, Y. S. y Guba, E. G. (1985). Naturalistic inquiry. Newbury Park: Sage.

McKernan, J. (1996). Investigación acción y curriculum. Madrid: Morata.

Mercer, N. y Fisher, E. (1992). How do teachers help children to learn? An analysis of teachers' interventions in computer-based tasks. Learning and Instruction, 2(4), 339-355.

https://doi.org/10.1016/9059-4752(92)90022-6

Metz, K. E. (2004). Children's understanding of scientific inquiry. Their conceptualization of uncertainty in investigations of their own design. Cognition and Instruction, 22(2), 219-290.

https://doi.org/10.1207/51532690xci2202_3

Oliva, J. M. (2019). Distintas acepciones para la idea de modelización en la enseñanza de las ciencias. Enseñanza de las Ciencias, 37(2), 5-24.

https://doi.org/10.5565/rev/ensciencias.2648 
Reigosa, C. E. y Jiménez, M. P. (2000). La cultura científica en la resolución de problemas en el laboratorio. Enseñanza de las Ciencias, 18(2), 275-284.

Richmond, G. (1998). Scientific apprenticeship and the role of public schools: general education of a better kind. Journal of Research in Science Teaching, 35(6), 583-587. https://doi.org/10.1002/(SICI)1098-2736(199808)35:6<583::AID-TEA1>3.0.CO;2-I

Roth, W.-M. y Bowen, G. W. (1995). Knowing and interacting: a study of culture, practices and resources in a grade 8 open-inquiry science classroom guided by a cognitive apprenticeship metaphor. Cognition and Instruction, 13(1), 73-128. https://doi.org/10.1207/s1532690xci130_3

Sandoval, W. A. y Morrison, K. (2003). High school students' ideas about theories and theory change after a biological inquiry unit. Journal of Research in Science Teaching, 40(4), 369-392. https://doi.org/10.1002/tea.10081

Stake, J. E. y Mares, K. R. (2001). science enrichment programmes for gifted high school girls and boys: predictors of programme impact on science confidence and motivation. Journal of Research in Science Teaching, 38(10), 1065-1088. https://doi.org/10.1002/tea.10001

Toplis, R. (2007). Evaluating science investigations at ages 14-16: dealing with anomalous data. International Journal of Science Education, 29(2), 127-150. https://doi.org/ 10.1080/095006690500498278

Wells, G. (1999). Dialogic inquiry. Toward a sociocultural practice and theory of education. Cambridge: Cambridge University Press.

Wood-Robinson, V., Watson, R. y Goldsworthy, A. (2000). Improving investigations from the AKSIS Project. Education in Science, 188, 14-15. 


\title{
Functionality of relationships among concepts for problem solving
}

\author{
Carlos Emilio Reigosa Castro \\ IES Lucus Augusti y Facultad de Formación del Profesorado (Lugo, España) \\ carlosreigosa@edu.xunta.gal
}

Here is presented a case study analyzing how the semantic relations among concepts are continuously transformed, refined and used by a group of $12^{\text {th }}$ grade students while solving tasks conceived as problems in the Physics laboratory.

The data consist of video and audio recordings of the students' verbal and physical actions during the sessions devoted to the tasks. The researcher was the students' teacher, so the process was developed as an action research experience, done with the intrinsic goal of improving the achievement of the students, and with the extrinsic goal of creating meanings useful for the science education community.

In the first task, the students had to decide the type of motion followed by a ball in a rail in both horizontal and inclined positions, and, in the second one, they had to choose between several springs which was the better one to weight little objects.

In the analysis, all the recordings were transcribed, and analysed using Lemke (1997) notion of contextualyzing practices. Following this theoretical perspective, here is considered that the participants' actions achieve meaning as they are connected to contexts. One type of context are the thematic patterns, which are the ways in which the members of a community speak about a specific subject. In a thematic pattern, the semantic relationships between concepts are continuously rebuilt.

The research question was: how do the students build semantic relationships between concepts, transforming them in functional tools to face open tasks in the laboratory?

The results show that the semantic patterns of each task are developed by the students through a laborious process. The actions are significant and useful only when they are placed in an adequate context, and here the students do not have such context at the beginning, so initially they are not able to solve the problems. We found that the semantic relationships among concepts go through a qualitative transformation with phases whose nature depends on the task and the resources displayed by the students.

In the first task, the students begin with disconnected concepts, which they connect later and then they use it as tools to take decisions, although initially these tools lack of functionality due to epistemological obstacles. And when they study the movement in the inclined rail, there are difficulties, in this case of procedural kind, due to aspects related to the control of the experimental error.

In the second task, they use, from the beginning, the relevant concepts as a context to take decisions, although initially these decisions do not have utility for the general objective of the task. After that, there are changes in the way they see the relationships among the related concepts, but the final solution they give is not completely satisfactory from a scientific point of view. In this group, it could be seen that the use they did of Hooke's Law inhibited the reasoning instead of promoting it.

To conclude, the results show that to develop an adequate semantic pattern to be used properly to solve a task can be a difficult and laborious process. But not only are the relationships among concepts essential, but also some obstacles of epistemological and procedural kinds, which limit the students' achievements, can be important.

And, finally, another conclusion of the article is the importance of the interaction with the teacher. Here is shown that one way for this interaction to be useful is to conceive it as scaffolding, allowing the students to achieve a higher competence level helping them to evoke useful contexts to connect their actions in a way that is helpful to solve each task. 
